\title{
Issues of transport security and human factor
}

\author{
Victoria Lez'er $^{1}$, Irina Muratova $^{1}$, and Natalia Korpusova ${ }^{1, *}$ \\ ${ }^{1}$ Tyumen Industrial University, Volodarskogo str., 38, Tyumen, 625000, Russia
}

\begin{abstract}
The article discusses the concept of human factor. The authors have studied the problem of transport security that depends on the sociopsychological component of the staff. The reasons for the significant influence of the human factor in the field of motor transport are identified, as well as the ways of formation of individual indicators in the structure of the emotional stability of the driver or conductor. The textual and graphical materials of the reports on the formation of a safety culture in transport organizations, as well as informational tables on the results of staff surveys, served as an empirical base. The study was made in support of the methods of cognitive modeling, questionnaires, application of the SHELL model, which reveals the main elements of the human factor and the relationship between them. Empirical Base - Tyumen Passenger Transport Organizations - DEP Tyumenavtotrans, individual entrepreneur Evrobus 72, Intellnvest LLC.
\end{abstract}

\section{Introduction}

The relevance of the stated research topic is explained by the fact that man-made accidents and disasters occur every day in the world; their causes should be sought in the specifics and characteristics of the human factor. According to the Health Organization, annually in the world only 1.2 million people die as a result of road accidents. According to the A. A. Blagonravov Institute of Mechanical Engineering of the Russian Academy of Sciences, the indicator of the importance of the human factor in major accidents at technical facilities in various areas of the technosphere in coefficient terms is the following: military aviation - 0.85 ; road transport - 0.80 ; civil and industrial construction - 0.70 ; civil aviation 0.65 ; nuclear power - 0.55 ; technological equipment -0.40 ; for military space technology 0.35 ; pipeline transport - 0.30 [1-3]. As we see, from the indicated technogenic spheres the overwhelming majority are interconnected with transport systems.

According to the analysis, the main factor of technogenic disasters and accidents are most often employees who violate established vehicle operation standards and safety regulations, or do not meet the proper qualifications or are not professionally able to solve transport tasks due to any physical quality, individually - psychological or social characteristics.

Statistical indicators indicate an insignificant role of violations in the production of vehicles, elements of infrastructure or design. A more significant factor in the practice of

\footnotetext{
*Corresponding author: a.copytowa@yandex.ru
} 
accidents is the mistakes of drivers and dispatchers. In the field of motor transport, the human factor causes $70-90 \%$ of all emergencies. Moreover, in $45-55 \%$ of accidents, personnel error is practically the only reason that leads to an accident. Technical malfunction is a major factor in only $4-5 \%$ of all emergencies, and the various circumstances of an unfavorable environment constitute only 5 to $10 \%$ of car accidents.

A similar pattern is noted by security experts from other countries, emphasizing the leading role of the human factor. Some researchers associate accidents with the negligence and inattention of staff from 60 to $90 \%$ of cases [4]. There are statistics that indicate a higher percentage of human guilt in accidents and disasters - from 95 to $99 \%$ [5].

The human factor is the cause of the termination or disruption of the functioning of a technical object, consisting of the organizational (nature of human relations) and individual-personal (human behavior) component.

The concept of "human factor" is a complex definition and includes several aspects determined by the specifics of professional activity. As for the sphere of transport, here the term "human factor" means a set of biological and physiological features of the body, as well as the socio-psychological characteristics of an employee, determining the level of his professional suitability, health, willingness to perform professional activities, the motivational component, degree of efficiency, degree of efficiency and psychological stability (V. Ponomarenko, 2004; V. Zvonikov, 2004; A. Vorona, 2001, etc.).

The socio-psychological component of the staff is of particular importance in the problem of transport security. In the context of this study, the theory of P.B. Zilberman, who interprets the emotional stability of an employee, not as the stability of emotional experiences, but as "... an integrative personality trait characterized by such an interaction of emotional, volitional, intellectual and motivational components of an individual's mental activity that ensure optimal successful achievement of the goal of activity in a complex emotive environment» [6].

The purpose of the study is to identify the causes of the significant influence of the human factor in the field of motor transport, as well as to determine the methods of formation of individual indicators in the structure of the emotional stability of the driver or conductor. A sample of 198 employees in the transport sector of the city of Tyumenwas made. The sample is random, homogeneous, which is evidence of its representativeness.

Used sources and empirical base

The theoretical basis of the research was the work of Maltsev M.V., Savchenko M.V., Karetnikova V.V., Kozik S.V., Sokolova I.A., Shevchenko A.I., Marinova M.L., Kaminsky V. Yu., Tomilina A.N., Tuktarova R.R., Bularova V.M., Gumerova K.M., Bochkareva T.S., Malinovsky, defining the main aspects of the problem of the influence of the human factor in the modern transport sector.

The following legislative and regulatory documents were used: "Labor Code of the Russian Federation” dated December 30, 2001 No. 197-FZ; .GOST P ISO 9001; GOST R ISO 26000-2010; ISO 14001.

Analytical reports of VTSIOM OJSC, EMERCOM of Russia.

The textual and graphical materials of the reports on the formation of a safety culture in transport organizations, as well as informational tables on the results of staff surveys, served as an empirical base.

The study was made in support of the methods of cognitive modeling, questionnaires and the personal questionnaire of the MLE-AM A.G. Maklakova and S.V. Cheryatyatina, application of the SHELL model, revealing the main elements of the human factor and the relationship between them.

To study the characteristics of the driver's personality, the 16-factor Cattel personal questionnaire (a comprehensive assessment of the personality) was used as the baseline. 
Empirical Base - Tyumen Passenger Transport Organizations - DEP Tyumenavtotrans, Individual entrepreneur Evrobus 72, IntelInvest LLC.

\section{Methods}

1. The study of driver stress tolerance

As a methodological base for the study of the formation of indicators of emotional sustainability, we used the method of questioning based on the personal questionnaire of MLE-AM A. G. Maklakova and S.V. Chermyatina. The questionnaire included 165 questions and the following measurement indicators: AA - adaptive abilities; NPS - neuropsychological stability; CF - communicative features; MS is for moral standard; A authenticity.

2. Cognitive modeling techniques

American cybernetic Marvin Minsky has developed an effective method of psychological and cognitive modeling, representing a systematic, orderly representation of human knowledge, in the form of pyramidal structures - frames, convenient for presenting these stereotypical situations.

Common with the frame type of knowledge representation systems are cognitive maps. This technique is used for cognitive modeling - processing and filtering incoming information [7] in cases when it is necessary to analyze the indefinite, inconsistent structures. In modern science, the technique of cognitive modeling is actualized in the practice of rationalization and improvement of the quality of research techniques and situation modeling.

With the help of a cognitive map, you can explore the cause-effect relationship of a particular area of knowledge, in the form of a directed graph. In formal terms, a cognitive map looks like the following ordered set of factors and relationships: $\mathrm{G}=\langle\mathrm{C}, \mathrm{W}\rangle$,

where $\mathrm{C}=\{\mathrm{c} 1, \mathrm{c} 2, \mathrm{c} 3 \ldots, \mathrm{cn}\}$ is a combination of factors acting as vertices of the graph and defined as concepts;

$\mathrm{W}=\{\mathrm{wij}=\mathrm{w}(\mathrm{ci}, \mathrm{cj})\}$ - a sequence of connections, defined as edges of a graph and connecting factors - concepts.

Therefore, the indicators ci and cj are in correlation with w. Ultimately, a change in the meaning of one connection - the cause - ci determines the change in the meaning of another connection - the effect - cj. If the values of the relationship-cause as a concept increases, then the result will be an increase in the value of the relationship-consequence with a simultaneous positive effect (cause-effect relationship) and a decrease in these values with a negative effect.

Cognitive modeling has a procedural nature, which is based on the following steps:

1.Formation of the methodological part of the study - definition of goals, objectives, main stages of modeling.

2. The study of a concrete, factual situation of the influence of the human factor in the aspect of the designated purpose. The collection, codification, analysis of the obtained quantitative or qualitative information is carried out, the necessary requirements, operating conditions, and the main limitations are characteristic of this technical system.

3. Analysis of direct and indirect factors that determine the main stages of the development of the studied situation or indirectly affect its changes. These include, first of all, targeted, motivational factors initially taken into account and directly influencing the result, as well as intermediate, bifurcation factors which influence was not previously taken into account.

4. Determination of the interdependence of various factors, their relations, directions, possible development options.

5. Formation of a cognitive map. 
6. Analysis of the efficiency or weight of the interdependence of various factors.

7. Verification of the correctness and effectiveness of the generated model: using the method of comparing the data obtained in an experiment with the actual, initial data of the problem situation.

8. Formulation of conclusions and recommendations taking into account the methodological part of the study indicated at the first stage.

The final stage of the universal cognitive map is the development of a plan of corrective measures to reduce the influence of the human factor in the field of road transport, provided that anthropological and technical resources are saved.

The universality of the proposed model is determined by the leading role of man as the central link of the entire system and the generality of the characteristics constituting the human factor. Thus, the application of this model is correct and effective for analyzing the level of safety of all types of vehicles.

Analysis of the influence of the human factor on the functioning of a complex erganic system, based on a formalized approach, taking into account the overall performance indicators, allows you to define uniform objective standards for a safe environment and create a positive driver's image that inspires confidence among managers, colleagues, and consumers.

3. Formation of a model, which use helps to determine the objective, formal characteristics of the human factor in the field of motor transport in order to study its influence in emergency situations.

The use of cognitive modeling reveals the reasons for the significant role of the human factor in road transport.

The driver as a central link must be included simultaneously in several surrounding systems - vehicle, procedures, external environment, internal social environment (passengers, colleagues).

This situation is correctly represented by the SHELL Model, revealing the most significant characteristics of the human factor, their interactions and consequences [8].

The driver of the vehicle is the main link of this model, included in the work of several subsystems:

1. "Liveware-Hardware" - functioning provides the energy resources of the interaction between man and vehicle;

2."Liveware-Liveware" - the work of the subsystem is based on social connections between the leader and the subordinate, the driver and the passenger, colleagues;

3."Liveware-Software" - the driver's involvement in the regulatory relationship, fulfillment of instructions etc .;

4."Liveware-Environment" - a set of relations between the driver and the conditions of his work, the compliance of internal and external factors of the working space with his psycho-physiological features.

The use of the SHELL model is effective in order to improve the quality of driver work and the level of safety in the field of motor transport. SHELL model represents:

1. The areas with the highest risk associated with human factors;

2. Objective reasons for the influence of the human factor in emergency situations;

3. Basic methods for collecting the necessary statistical and qualitative information.

\section{Results}

1. Analyzing the data obtained as a result of testing by A.G. Maklakov [9] identified positive changes in the following personal characteristics of the drivers of the experimental group: neuro-psychological stability, adaptive abilities (Table 1), which indicates the need to improve measures of social and psychological training of employees. 
Table 1. Research results.

\begin{tabular}{|c|c|c|c|c|}
\hline \multirow{2}{*}{ Scale } & \multirow{2}{*}{ Group } & \multicolumn{3}{|c|}{ Levels } \\
\cline { 3 - 5 } & & High & Average & Low \\
\hline \multirow{2}{*}{ Neuropsychic stability } & EG & $69 \%$ & $31 \%$ & $0 \%$ \\
\cline { 2 - 5 } & CG & $58 \%$ & $32 \%$ & $10 \%$ \\
\hline \multirow{2}{*}{ Adaptive abilities } & EG & $78 \%$ & $21 \%$ & $1 \%$ \\
\cline { 2 - 5 } & CG & $71 \%$ & $20 \%$ & $9 \%$ \\
\hline
\end{tabular}

2. The results of research activities in the Tyumen passenger transport organizations DEP Tyumenavtotrans, Evrobus 72, IntelInvest LLC to minimize the risks of the human factor

In order to reduce the level of influence of the human factor in the Tyumen passenger transportation organizations - DEP Tyumenautotra, a program was developed aimed at developing a safety culture, increasing the level of personnel responsibility and eliminating manifestations of opportunism. For the development of corporate culture, a universal "road map" for the current year, effective emergency prevention measures and training sessions have been proposed. There is a program to improve corporate culture, which includes the following innovative measures.

I) The company conducts corporate certification based on stimulating the motivation of staff as a corporate community, whose work is determined by the synergistic effect (increase in business efficiency due to the system effect - emergence).

II) There is used coupon system for monitoring compliance with safety regulations. The perspective of this method is based on the development of corporate culture and personnel responsibility.

III) The program to improve the level of competence of employees includes innovative training programs for staff, training sessions using simulators and qualifying interviews.

IV) The development program of the motivational potential of employees of the enterprise increases the safety culture, based on the introduction of the principle of the active position of the employee, his involvement in the main corporate functions.

V) The program for the development of communication activities was introduced with the aim of expanding the practice of publishing activity of staff, exchanging experience, creating an information space between corporate media of the infrastructure complex. The main task is to describe the best practices in the specific cases of preventing violations of safety rules.

3. Taking the SHELL model as the main methodological toolkit, it is possible to determine the main statistical and qualitative characteristics of the human factor in the field of motor transport.

The "subject"-driver component of a model is considered its professional, anthropometric, physiological, socio-psychological characteristics that can be evaluated quantitatively (age, health category, weight, height) or quality indicators (skill level, amount of professional knowledge).

The "environmental" component includes the characteristics of the workspace itself (sound insulation, temperature, accessibility to view the road, etc.) and external environmental factors (weather conditions, road obstacles, time of day).

Component "equipment" includes vehicle characteristics, which are assessed either by quantitative indicators (lifetime) or qualitative (ergonomic).

The component "procedure" includes a documentation system and the practice of instructions, which are characterized quantitatively (number of pages, tables) and qualitatively (level of compliance with standards).

This model is effective in determining the level of significance of the considered components relative to the driver's activities. It is used as a system for assessing the level of 
compliance of the chosen profession with the individual characteristics of a particular person.

\section{Discussion}

The debatable problems in this area remain the search for ways to most effectively manage the ergatic system by the driver. The difficulty is that the driver, as the central link of the system, is simultaneously included in the work of several independent subsystems. Each subsystem, in turn, is interconnected by backward channels. Therefore, the driver of public transport must be in the same set not only with the internal environment, which represents his workplace, but also with the external environment — other cars, roads, weather conditions, etc.

Researchers continue to discuss what conditions must be met for the safe, efficient operation of this ergatic system. In particular, the following conditions are given for compatibility and effective interaction between the driver and the vehicle:

1. The compatibility of spatial-anthropometric characteristics includes indicators of compliance of the equipment of the workplace with the anthropometric features of the driver. For example, the compliance of the design of the accessibility zone for the limbs and the working position of the length of the limbs of the driver.

2. Biophysical compatibility is determined by the compliance of the improvement of the environment with the provision of the necessary working capacity and the maintenance of a healthy physical state of the body. These factors include temperature, atmospheric pressure, light, etc.

3. Energy compatibility is to ensure effective interaction between the driver and the vehicle. It is considered as the coordination, for example, of the optimal capabilities of the driver and the power of the vehicle; driver coordination and maximum vehicle speed.

4. Compatibility information is rated as the most popular in the field of modern vehicles. It lies in accordance with the existing information platform of the vehicle to the physiological characteristics of the driver. For the successful interaction of the driver and the vehicle, the ability of the information system to reflect the important characteristics of the equipment and indicators of its functioning at the required time is important. This type of compatibility allows the driver to correctly make decisions on how to drive a vehicle based on the information received on time. The solution of this task increases the efficiency of personnel work and, in general, the safety culture of the motor transport industry.

5. Technical and aesthetic compatibility is determined by the satisfaction of the driver's aesthetic need for the process of interaction with the vehicle, for example, at the expense of the make of the car, the design of the devices, etc.

Thus, the problem of transport safety is solved when the quality of the vehicle corresponds to the basic physiological, socio-psychological characteristics of the driver. At the same time, the interaction in the ergatic system is due to the mutual influence of man and machine, the exchange of their production functions. In this way, there is a mutual adaptation process that provides an opportunity to solve new problems, improve the level of driver skill and safety of vehicles.

\section{Conclusion}

As shown by a study of the influence of the human factor on the functioning of transport systems, using the example of the Tyumen Passenger Transport Organization - DEP Tyumenautotra, there are many ways to effectively prevent technological accidents and 
reduce the level of potential threats associated with opportunistic behavior of personnel. These security resources can be classified for the following reasons.

Organization of external security resources.

- control over information resources: control of the volume, complexity, speed, form of presentation of information;

- organization and management of hardware: consideration of the compatibility of the characteristics of the workplace and the characteristics of the driver as the central link of the ergatic system;

- ensuring the conditions for effective human interaction with software systems: consideration of a favorable user interface;

- provision of comfortable working conditions for the driver: the formation of conditions that allow to observe the regime of work and rest.

Organization of internal security resources.

- improving the quality of professional selection for the designated qualification criteria: professional competence, stability of the nervous system, responsibility, working capacity etc.

- organization of training and driver development: providing modern scientific literature, conducting professional trainings, training on simulators, etc.

Organization of psychological security support resources.

- determination of the level of staff motivation to form a safety culture;

- improving the corporate culture to ensure a positive socio-psychological climate.

Thus, the driver's activity is fraught with enormous physical, social, and moralpsychological risks. This requires a high level of professional skills, good health, resistance to the effects of many internal and external negative environmental factors. Only people who are professionally competent, healthy, physically fit, with a high level of responsibility and emotional stability are able to safely perform the work of a driver of public urban transport.

\section{Acknowledgement}

The work was supported by Act 211 of Government of the Russian Federation, contract No 2 A03. 2111

\section{References}

1. A. Truntsevsky, Y.V. Lukiny, I.I. Sumachev, A.V. Kopytova, MATEC Web of Conferences 170, 01067 (2018) DOI: 10.1051/matecconf/201817001067

2. N. Zotkina, S. Bardasov, M. Gusarova, A. Kopytova, MATEC Web of Conferences 106, 08050 (2017) DOI: 10.1051/matecconf/201710608050

3. B.S. Dobroborsky, Machine Safety and Human Factors (SPbGASU Publishing House, $\mathrm{SPb}, 2011)$

4. I.A. Vaseltsova, Scientific notes of the PF University, Lesgaft 12(46), 7-11 (2008)

5. B. Kosko ,International Journal of Man-Machine Studies 1, 65-75 (1986)

6. A.G. Maklakov, Psychological journal 22(1), 16-24 (2001)

7. M. Gravit, O. Zybina, A. Vaititckii, A. Kopytova, IOP Conference Series: Earth and Environmental Science 90 (1), 012103 (2017) DOI: 10.1088/1755-1315/90/1/012103

8. A. Kopytova, MATEC, 106, 08056 (2017) DOI: 10.1051/matecconf/201710608056

9. A.V. Kopytova, Exchange of intellectual property 3(XIV), 31-37 (2015) 